To V.P. Havin on the occasion of his 75th birthday

\title{
A BLASCHKE-TYPE CONDITION FOR ANALYTIC AND SUBHARMONIC FUNCTIONS AND APPLICATION TO CONTRACTION OPERATORS
}

\author{
S. FAVOROV AND L. GOLINSKII
}

\begin{abstract}
Let $E$ be a closed set on the unit circle. We find a Blaschke-type condition, optimal in a sense of the order, on the Riesz measure of a subharmonic function $v$ in the unit disk with a certain growth at the direction of $E$. In particular case when $E$ is a finite set, and $v=\log |f|$ with an analytic function $f$, our result agrees with the recent one by A. Borichev, L. Golinskii and S. Kupin. An application to contractions close to unitary operators in the Hilbert space is given.
\end{abstract}

\section{InTRODUCTION}

In [1] the authors study zero sets of analytic functions in the unit disk $\mathbb{D}$, which grow exponentially fast near a finite set $E$ of points on the unit circle $\mathbb{T}$. Here is the main result of [1]. As usual, $x_{+}=\max \{x, 0\}$.

Theorem BGK. Let $E \subset \mathbb{T}$ be a finite set, $f \in \mathcal{A}(\mathbb{D})$ an analytic function in $\mathbb{D},|f(0)|=1$, and

$$
|f(z)| \leq \exp \left(\frac{D}{\rho^{q}(z, E)}\right)
$$

with $D, q \geq 0, \rho(z, E)=\operatorname{dist}(z, E)$. Let $Z_{f}$ be the zero set of $f$, each zero is counted according to its multiplicity. Then for any $\varepsilon>0$,

$$
\sum_{z \in Z_{f}}(1-|z|) \rho(z, E)^{(q-1+\varepsilon)_{+}} \leq C(\varepsilon, q, E) D .
$$

Note that both (1) and (2) make sense for arbitrary (infinite) subsets $E$ on $\mathbb{T}$, so the question arises naturally, whether the finiteness of $E$, which is an essential ingredient in the proof of Theorem BGK, can be relaxed. We should also mention that in the case $E=\mathbb{T}$, $\rho(z, E)=1-|z|$ we come to the well known Blaschke-type condition

1991 Mathematics Subject Classification. Primary: 30D50; Secondary: 31A05, $47 \mathrm{~B} 10$.

Key words and phrases. analytic function in the unit disk, subharmonic function in the unit disk, Riesz measure, Blaschke-type condition, discrete spectrum, contraction operator. 
for analytic functions in $\mathbb{D}$ with radial growth. The theory of such functions goes back to M.M. Djrbashian [4], see also V.I. Matsaev \& E.Z. Mogulskii [16], W. Hayman \& B. Korenblum [11], F.A. Shamoyan [18], A.M. Jerbashian [13].

Our investigation shows that the natural setting for the above problem is the class of subharmonic functions $v$ and their Riesz measures (generalized Laplacians) $\mu=(1 / 2 \pi) \Delta v$ rather than analytic functions and their zero sets. It turns out that arbitrary closed sets $E$ can be involved, and the results are optimal. Note that in the case of subharmonic functions of the form $\log |f|$ with $f \in \mathcal{A}(\mathbb{D})$, the Riesz measure is a discrete and integer-valued measure supported on $Z_{f}$, and $\mu\{z\}$ equals the multiplicity of zero of $f$ at $z$.

To formulate our result we need the following quantitative characteristic of the "sparseness" of a closed set $E$ in terms of its $t$-neighborhood

$$
E_{t}:=\{\zeta \in \mathbb{T}: \rho(\zeta, E)<t\} .
$$

Put

$$
I(\alpha, E):=\int_{0}^{2} \frac{\nu_{E}(s)}{s^{\alpha+1}} d s \leq+\infty, \quad \nu_{E}(t)=\left|E_{t}\right|, \quad \alpha \in \mathbb{R} .
$$

Here and in what follows we denote by $|A|$ the normalized Lebesgue measure of a set $A \subset \mathbb{T}$. It is not hard to see that $I(\alpha, E)<\infty$ for any $E$ and an arbitrary negative $\alpha$. In the case when $\nu_{E}(t)=O\left(t^{\beta}\right), \beta>0$, one has $I(\alpha, E)<\infty$ for $\alpha<\beta$. Examples of sets $E$ and evaluations of their characteristic $I$ are given in Section 2 .

The main result of our paper is the following

Theorem 1. Let $E \subset \mathbb{T}$ be a closed set. Let $v$ be a subharmonic function in $\mathbb{D}, v \neq \equiv-\infty, \mu$ its Riesz measure, and for all $z \in \mathbb{D}$ and some $q>0$

$$
v(z) \leq \frac{1}{\rho^{q}(z, E)}
$$

(i) If $I(q, E)<\infty$, then

$$
\int_{\mathbb{D}}(1-|\lambda|) d \mu(\lambda)<\infty .
$$

Moreover, if $v(0) \geq 0$, then

$$
\int_{\mathbb{D}}(1-|\lambda|) d \mu(\lambda) \leq 1+q 2^{q} I(q, E) .
$$

(ii) If $I(q, E)=\infty$ and $I(\alpha, E)<\infty$ for some $\alpha<q$, then

$$
\int_{\mathbb{D}}(1-|\lambda|)(\rho(\lambda, E))^{q-\alpha} d \mu(\lambda)<\infty .
$$

Moreover, if $v(0) \geq 0$, then

$$
\int_{\mathbb{D}}(1-|\lambda|)(\rho(\lambda, E))^{q-\alpha} d \mu(\lambda)<36\left(2^{-\alpha}+q(90)^{q-\alpha} I(\alpha, E)\right) .
$$


The result turns out to be optimal in the following sense.

Theorem 2. Let $E \subset \mathbb{T}$ be a closed set so that $I(\alpha, E)=+\infty$ for some $\alpha \geq 0$. Then for the subharmonic function $v_{0}(z)=\rho^{-q}(z, E)$ with the Riesz measure $\mu_{0}$, and $q \geq \alpha$, we have

$$
\int_{\mathbb{D}}(1-|\lambda|)(\rho(\lambda, E))^{q-\alpha} d \mu_{0}(\lambda)=+\infty .
$$

As a consequence we obtain

Theorem 3. Let $E \subset \mathbb{T}$ be a closed set, and $I(\alpha, E)<\infty$ for some $\alpha \in \mathbb{R}$. Let $f \in \mathcal{A}(\mathbb{D})$, and

$$
|f(z)| \leq \exp \left(\frac{D}{\rho^{q}(z, E)}\right), \quad z \in \mathbb{D} .
$$

Then

$$
\sum_{z \in Z_{f}}(1-|z|) \rho(z, E)^{(q-\alpha)_{+}}<\infty .
$$

Moreover, if $|f(0)| \geq 1$, then

$$
\sum_{z \in Z_{f}}(1-|z|) \rho(z, E)^{(q-\alpha)_{+}} \leq C(q, \alpha, E) D
$$

$C(q, \alpha, E)$ does not depend on $f$. In the case $q>\alpha$ one can take $C(q, \alpha, E)=36\left(2^{-\alpha}+q(90)^{q-\alpha} I(\alpha, E)\right)$.

The classical Blaschke condition arises for $E=\mathbb{T}, \alpha=-q<0$ and $q \rightarrow 0$.

The first statement of Theorem 3 is a certain uniqueness theorem: under assumption (10) the divergence of (11) yields $f \equiv 0$.

We proceed as follows. In Section 2 the main results are proved. In Section 3 an application to the spectral theory of contractions in the Hilbert space is discussed.

\section{Proof of THE MAIN RESUlts}

There is a simple way to compute the function $\nu_{E}$ defined in (4), in terms of the complimentary arcs of $E .{ }^{1}$ Let

$$
\mathbb{T} \backslash E=\bigcup_{j} \gamma_{j}, \quad\left|\gamma_{j}\right| \downarrow 0 .
$$

Then

$$
\nu_{E}(t)=\sum_{j=N+1}^{\infty}\left|\gamma_{j}\right|+\frac{2 N}{\pi} \arcsin \frac{t}{2}+|E|,
$$

where $N=N(t)$ is taken from

$$
\left|\gamma_{N+1}\right| \leq \frac{2}{\pi} \arcsin \frac{t}{2}<\left|\gamma_{N}\right|
$$

\footnotetext{
${ }^{1} \mathrm{An}$ interesting case occurs when the number of complimentary arcs is infinite.
} 
So the exponent $\alpha$ in (4), for which $I(\alpha, E)<\infty$ can be easily determined. For instance, for

$$
E=\left\{e^{i \varphi_{n}}: \varphi_{n}=\sum_{k=n}^{\infty} \frac{1}{2^{k}}\right\} \cup\{1\}
$$

one can take any $\alpha<1$, for

$$
E=\left\{e^{i \varphi_{n}}: \varphi_{n}=c \sum_{k=n}^{\infty} \frac{1}{k^{\gamma}}, \quad \gamma>1\right\} \cup\{1\}, \quad c^{-1}=\sum_{k=1}^{\infty} \frac{1}{k^{\gamma}},
$$

any $\alpha<1-\frac{1}{\gamma}$ works, for

$$
E=\left\{e^{i \varphi_{n}}: \varphi_{n}=c \sum_{k=n}^{\infty} \frac{1}{k \log ^{2} k}\right\} \cup\{1\}, \quad c^{-1}=\sum_{k=2}^{\infty} \frac{1}{k \log ^{2} k},
$$

$I(\alpha, E)<\infty$ for any $\alpha<0$. For the generalized Cantor set $\mathcal{C}_{\beta}$ (for the standard Cantor set $\beta=1 / 3)$ one can take any $\alpha<1-d(\beta)$,

$$
d(\beta)=\frac{\log 2}{\log 2-\log (1-\beta)}
$$

is the Hausdorff dimension of $\mathcal{C}_{\beta}$ (see, e.g., [6]).

Let us list some elementary properties of $\nu_{E}$.

(1) $\nu_{E}$ is a continuous and strictly monotone increasing function on $\left[0, t_{0}(E)\right]$ and $\nu_{E}(t)=1$ for $t_{0}(E) \leq t<\infty$;

(2) $\nu_{E}(0+)=|E|$

(3) $\nu_{E}(t) \geq|E|+t / \pi$ for each closed $E \neq \mathbb{T}$ and $0<t \leq t_{1}(E)$;

(4) $\nu_{E}(t)=O(t)$, as $t \rightarrow 0$, if and only if $E$ is a finite set.

Our argument relies upon some basic results from the potential theory in the complex plane (see $[17$, Chapters 3,4$]$ ). Let $v$ be a subharmonic function on $\mathbb{D}, v \not \equiv-\infty$. The Riesz measure $\mu=(1 / 2 \pi) \triangle v$ is known to be a positive Radon measure on $\mathbb{D}$. If $v$ has a harmonic majorant on a subdomain $\Omega \subseteq \mathbb{D}$, then the following representation holds

$$
v(z)=u(z)-\int_{\Omega} G_{\Omega}(z, \lambda) d \mu(\lambda), \quad z \in \Omega,
$$

where $u$ is the least harmonic majorant in $\Omega$, and $G_{\Omega}(z, \lambda)$ is Green's function for $\Omega$, that is,

$$
G_{\Omega}(z, \lambda)=\log (1 /|z-\lambda|)-h(z, \lambda),
$$

$h(z, \cdot)$ is a harmonic function in $\Omega$ such that $h(z, \lambda)=\log (1 /|z-\lambda|)$ for $\lambda \in \partial \Omega$. Note that if $\Omega \neq \mathbb{D}$ and $0 \in \Omega$, then $h(0, \lambda)>0$ for $\lambda \in \Omega$.

Proof of Theorem 1. We begin with a proof of (6). It easily follows from $I(q, E)<\infty, q>0$, and (4) that now $\nu_{E}(0+)=|E|=0$. Put $\rho(z)=\rho(z, E)$. 
The monotone permutation theorem (also known as the "layer cake representation", see, e.g., [15, Theorem 1.13])

$$
\int_{X} f^{r}(x) d \sigma(x)=r \int_{0}^{\infty} y^{r-1} \sigma(\{x: f(x)>y\}) d y
$$

is of importance in our argument. Here $r>0,(X, \sigma)$ is a measure space, $f \geq 0$ a measurable function on $X$. We have (see (4))

$$
\begin{aligned}
\int_{\mathbb{T}} \frac{d m(\zeta)}{\rho^{q}(\zeta)} & =q \int_{0}^{\infty} y^{q-1}\left|\left\{\zeta: \rho(\zeta)<\frac{1}{y}\right\}\right| d y \\
& =2^{-q}+q I(q, E)<\infty
\end{aligned}
$$

$d m$ is the normalized Lebesgue measure on $\mathbb{T}$. Therefore the Poisson integral

$$
U(z)=\int_{\mathbb{T}} \frac{1-|z|^{2}}{|\zeta-z|^{2}} \frac{d m(\zeta)}{\rho^{q}(\zeta)}, \quad z \in \mathbb{D}
$$

is a well defined harmonic function.

Let us prove that $v$ admits a harmonic majorant on the whole disk $\mathbb{D}$. A simple geometrical inequality shows that for all $|z| \leq 1$ and $0<\tau<1$ one has $\rho(z) \leq 2 \rho(\tau z)$. The function $v_{0}=\rho^{-q}$ is obviously subharmonic in $\mathbb{D}$ since

$$
v_{0}(z)=\sup _{\zeta \in E}\left(|z-\zeta|^{-q}\right)
$$

(see [17, Theorem 2.4.7]), and continuous at any point $\zeta \in \mathbb{T} \backslash E$. By the well known property of the Poisson integral

$$
\lim _{z \rightarrow \zeta} U(z)=v_{0}(\zeta) \geq 2^{-q} v_{0}(\tau \zeta), \quad \zeta \in \mathbb{T} \backslash E, \quad 0<\tau<1
$$

As long as $\zeta \in E$, one has $\lim _{z \rightarrow \zeta} U(z)=+\infty$. But $v_{0}(\tau z)$ is a bounded subharmonic function in $\mathbb{D}$, so by the Maximum Principle $U(z) \geq 2^{-q} v_{0}(\tau z), z \in \mathbb{D}$. It remains only to tend $\tau \rightarrow 1$ to make sure that $2^{q} U$ is a desired harmonic majorant for $v$.

Representation (12) takes now the form

$$
v(z)=u(z)-\int_{\mathbb{D}} \log \left|\frac{1-\bar{\lambda} z}{z-\lambda}\right| d \mu(\lambda), \quad z \in \mathbb{D},
$$

where $u \leq U$ in $\mathbb{D}$. For $z=0$ we come to

$$
\int_{\mathbb{D}}(1-|\lambda|) d \mu(\lambda) \leq \int_{\mathbb{D}} \log \frac{1}{|\lambda|} d \mu(\lambda) \leq u(0) \leq 2^{q} U(0)=2^{q}\left\|\rho^{-q}\right\|_{L^{1}(\mathbb{T})},
$$

and (6) follows from (15).

The case when $I(q, E)=\infty$ but $I(\alpha, E)<\infty$ for some $\alpha<q$ is much more delicate. 
For fixed $t \in(0,1)$ denote by $\Omega=\Omega_{t}$ the connected component of the open subset $\{z \in \mathbb{D}: \rho(z)>t\}$, which contains the origin, so $\Omega$ is a nonempty subdomain of $\mathbb{D}$. Put

$$
\Gamma:=\{z \in \mathbb{D}: \rho(z)=t\}, \quad E_{t}^{c}=\mathbb{T} \backslash E_{t},
$$

$E_{t}$ is defined in (3). It is easy to check that $E_{t}$ is a finite union of disjoint open arcs, so $E_{t}^{c}$ is a finite union of disjoint closed arcs. The boundary $\partial \Omega$ is contained in the closure of $E_{t}^{c} \cup \Gamma$. The function $v$ is bounded from above in $\Omega$, so (12) holds for $v$ in $\Omega$, with the least harmonic majorant $u$.

Let $V$ be a harmonic function in $\mathbb{D}$ such that

$$
V(\zeta)= \begin{cases}t^{-q}, & \zeta \in E_{t} \\ \rho^{-q}(\zeta), & \zeta \in E_{t}^{c}\end{cases}
$$

Note that $V$ is continuous in the closed unit disk. For $0<t<1$ and each $z \in \Gamma$ there is $\zeta^{\prime} \in E$ such that $\left|z-\zeta^{\prime}\right|=t$. Let $\omega\left(\lambda, \gamma_{z}, \mathbb{D}\right)$ be the harmonic measure of the arc $\gamma_{z}=\left\{\zeta \in \mathbb{T}:\left|\zeta-\zeta^{\prime}\right| \leq t\right\}$. It easily follows from the explicit expression (see, e.g., [7, Chapter 1]) that $\omega\left(\lambda, \gamma_{z}, \mathbb{D}\right) \geq 1 / 6$ for $\left|\lambda-\zeta^{\prime}\right|=t, \lambda \in \mathbb{D}$. Since $\gamma_{z} \subset E_{t}$, we get $V(z) \geq t^{-q} \omega\left(z, \gamma_{z}, \mathbb{D}\right) \geq t^{-q} / 6$. Therefore, we have

$$
\limsup _{z \rightarrow \zeta} v(z) \leq 6 V(\zeta), \quad \forall \zeta \in \partial \Omega .
$$

By the Maximum Principle

$$
v(z) \leq 6 V(z) \quad \text { and so } \quad u(z) \leq 6 V(z), \quad z \in \Omega .
$$

Let us prove (8). Since $v(0) \geq 0,(12)$ for $z=0$ can be written as

$$
\int_{\Omega} G_{\Omega}(0, \lambda) d \mu(\lambda) \leq u(0) \leq 6 V(0)=\frac{6 \nu_{E}(t)}{t^{q}}+6 \int_{E_{t}^{c}} \frac{d m(\zeta)}{\rho^{q}(\zeta)} .
$$

We proceed with another application of (14) to obtain

$$
\begin{aligned}
\int_{E_{t}^{c}} \frac{d m(\zeta)}{\rho^{q}(\zeta)} & =q \int_{0}^{\infty} y^{q-1}|\{\zeta \in \mathbb{T}: t<\rho(\zeta)<1 / y\}| d y \\
& =2^{-q}-\frac{\nu_{E}(t)}{t^{q}}+q \int_{t}^{2} \frac{\nu_{E}(s)}{s^{q+1}} d s .
\end{aligned}
$$

Hence, the following bound holds

$$
\int_{\Omega} G_{\Omega}(0, \lambda) d \mu(\lambda) \leq 6 \cdot 2^{-q}+6 q \int_{t}^{2} \frac{\nu_{E}(s)}{s^{q+1}} d s .
$$

Our next step is to obtain a lower bound for the Green function $G_{\Omega}(0, \cdot)$ in a smaller domain $\Omega_{\tau}$ with $\tau=(6 \pi+3) t$. We assume now that $t \in\left(0, \frac{1}{6 \pi+3}\right)$, and so $\tau \in(0,1)$. For $z \in \Gamma$ there is $\zeta^{\prime} \in E$ such that $\left|z-\zeta^{\prime}\right|=t$, so $t \geq 1-|z|$, and hence

$$
\log \frac{1}{|z|} \leq \log \frac{1}{1-t} \leq \frac{3 t}{2}
$$


The harmonic function $h(0, \cdot)$ from $(13)$ does not exceed $3 t / 2$ on $\Gamma$ and equals zero on $E_{t}^{c}$, so

$$
h(0, \lambda) \leq \frac{3 t}{2}, \quad \lambda \in \Omega
$$

We will distinguish two situations for $\lambda$.

Let $|\lambda| \leq 1-2 t$. Then by $(20)$

$$
G_{\Omega}(0, \lambda)=\log \frac{1}{|\lambda|}-h(0, \lambda) \geq 1-|\lambda|-\frac{3 t}{2} \geq \frac{1-|\lambda|}{4} .
$$

Let $|\lambda|>1-2 t$. This is where the restriction $\lambda \in \Omega_{\tau}$ is essential. Let $\omega\left(\lambda, E_{t}, \mathbb{D}\right)$ be the harmonic measure of the set $E_{t}$. We put $g(\lambda)=$ $9 \operatorname{t\omega }\left(\lambda, E_{t}, \mathbb{D}\right)$. The same argument as above implies $\omega\left(\lambda, E_{t}, \mathbb{D}\right) \geq 1 / 6$ for $\lambda \in \Gamma$, so

$$
h(0, \lambda) \leq g(\lambda), \quad \lambda \in \partial \Omega \quad \Rightarrow \quad h(0, \lambda) \leq g(\lambda), \quad \lambda \in \Omega
$$

and

$$
G_{\Omega}(0, \lambda) \geq 1-|\lambda|-g(\lambda) .
$$

We will find the upper bound for $g(\lambda)$ in $\Omega_{\tau}$. For $\lambda=|\lambda| e^{i \theta}$ write

$g(\lambda)=9 t \int_{E_{t}} \frac{1-|\lambda|^{2}}{|\zeta-\lambda|^{2}} d m(\zeta)=\frac{9 t\left(1-|\lambda|^{2}\right)}{2 \pi} \int_{e^{i \varphi \in E_{t}}} \frac{d \varphi}{(1-|\lambda|)^{2}+4|\lambda| \sin ^{2} \frac{\varphi-\theta}{2}}$.

Since $\rho(\lambda) \geq \tau$ and $1-|\lambda|<2 t$, we have for some $\zeta_{1} \in E$

$$
\begin{aligned}
\rho\left(e^{i \theta}\right) & =\left|e^{i \theta}-\zeta_{1}\right|=\left|\lambda-\zeta_{1}+e^{i \theta}-\lambda\right| \\
& \geq(6 \pi+3) t-(1-|\lambda|)>(6 \pi+1) t .
\end{aligned}
$$

Next, for $e^{i \varphi} \in E_{t}$ there is $\zeta_{2} \in E$ with $\left|e^{i \varphi}-\zeta_{2}\right|=\rho\left(e^{i \varphi}\right) \leq t$, so

$$
|\theta-\varphi| \geq 2\left|\sin \frac{\theta-\varphi}{2}\right|=\left|e^{i \theta}-\zeta_{2}+\zeta_{2}-e^{i \varphi}\right| \geq \rho\left(e^{i \theta}\right)-t \geq 6 \pi t .
$$

Therefore, as $|\lambda|>1-2 t>9 / 10$, we come to

$$
g(\lambda) \leq \frac{5 \pi t(1-|\lambda|)}{2} \int_{6 \pi t \leq|\varphi-\theta| \leq \pi} \frac{d \varphi}{(\varphi-\theta)^{2}}<\frac{5(1-|\lambda|)}{6} .
$$

Finally, the following lower bound holds for all $\lambda \in \Omega_{\tau}$

$$
G_{\Omega}(0, \lambda) \geq \frac{1-|\lambda|}{6}
$$

and we can continue (19) as

$$
\int_{\Omega_{\tau}}(1-|\lambda|) d \mu(\lambda) \leq 36\left(2^{-q}+q \int_{\tau /(6 \pi+3)}^{2} \frac{\nu_{E}(s)}{s^{q+1}} d s\right) .
$$

The latter holds for each $\tau \in(0,1)$.

Define $\tilde{\rho}:=\min \{\rho, 1\}$. It is easily checked that

$$
\tilde{\rho}(\lambda) \leq \rho(\lambda) \leq 2 \tilde{\rho}(\lambda) \quad \forall \lambda \in \mathbb{D},
$$


and $\{\lambda: \rho(\lambda)>\tau\}=\{\lambda: \tilde{\rho}(\lambda)>\tau\}$ for $0<\tau<1$. (14) applied to the measure $d \sigma=(1-|\lambda|) d \mu$ and the function $\tilde{\rho} \leq 1$ gives

$$
\int_{\mathbb{D}}(\tilde{\rho}(\lambda))^{q-\alpha} d \sigma(\lambda)=(q-\alpha) \int_{0}^{1} \tau^{q-\alpha-1} d \tau \int_{\{\rho>\tau\}} d \sigma(\lambda) .
$$

Since $\rho(z) \leq 2 \rho(\tau z)$ for $z \in \mathbb{D}$ and $0<\tau<1$ then $\rho(\tau z)>\tau / 2$, as long as $\rho(z)>\tau$, so the whole interval $[0, z]$ belongs to the set $\{\rho>\tau / 2\}$. The latter means that $\{\rho>\tau\} \subset \Omega_{\tau / 2}$. Hence by using (22) we have

$$
\int_{\mathbb{D}}(\rho(\lambda))^{q-\alpha} d \sigma(\lambda) \leq 2^{q-\alpha}(q-\alpha) \int_{0}^{1} \tau^{q-\alpha-1} d \tau \int_{\Omega_{\tau / 2}}(1-|\lambda|) d \mu(\lambda),
$$

and (21) leads to (8), as claimed.

To prove $(5),(7)$ in the case $v(0)>-\infty$, one can apply (6), (respectively, (8)) to the function $v_{1}(z)=(v(z)-v(0))\left(1+2^{q}|v(0)|\right)^{-1}$. Indeed, $v_{1}(z) \leq \rho^{-q}(z)$ for all $z \in \mathbb{D}$, and the Riesz measure of $v_{1}$ coincides with the Riesz measure of $v$ up to a constant factor (which depends on $v(0)$ ).

If $v(0)=-\infty$, take the harmonic function $h$ in the disk $\{|z|<1 / 2\}$ such that $v=h$ for $|z|=1 / 2$, and put

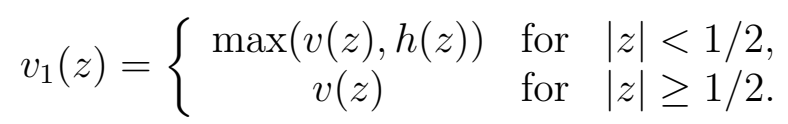

Clearly, $v_{1}(z) \leq v(z) \leq \rho^{-q}(z, E)$. Moreover, $v_{1}$ is subharmonic in $\mathbb{D}$ (see, e.g., [17, Theorem 2.4.5]), and the restriction of its Riesz measure $\mu_{1}$ on the set $\{z \in \mathbb{D}:|z|>1 / 2\}$ is the same as one for $\mu$. Therefore, the integrals in (5), (7) taken for $\mu$ and $\mu_{1}$ differ by a bounded term. Since $v_{1}(0) \neq \infty$, the result follows. The proof is complete.

Proof of Theorem 2. As it was mentioned above, the function $v_{0}=$ $\rho^{-q}$ is subharmonic in $\mathbb{D}$. We invoke again the harmonic function $V$ (16). It is clear that $V=v_{0}$ on $E_{t}^{c}$, and $V \leq t^{-q}=v_{0}$ on $\Gamma$. Put $u_{0}$ for the least harmonic majorant of $v_{0}$ on $\Omega=\Omega_{t}$, which is defined above. We have

$$
V(\zeta) \leq v_{0}(\zeta) \leq u_{0}(\zeta), \quad \zeta \in \partial \Omega \quad \Rightarrow \quad V(z) \leq u_{0}(z), \quad z \in \Omega .
$$

Since $h(0, \cdot) \geq 0$ and $v_{0}(0)=1$, then by $(12)$ for $z=0$

$$
V(0) \leq u_{0}(0) \leq \int_{\Omega} \log \frac{1}{|\lambda|} d \mu_{0}(\lambda)+1
$$

For $V(0)$ we see (cf. (17)-(18)) that

$$
V(0)=2^{-q}+q \int_{t}^{2} \frac{\nu_{E}(s)}{s^{q+1}} d s .
$$

Thereby, in the case $q=\alpha$ we have

$$
\int_{\mathbb{D}} \log \frac{1}{|\lambda|} d \mu_{0}(\lambda)=+\infty
$$


Let now $q>\alpha$. We apply (14) with $d \sigma=\log |\lambda|^{-1} d \mu_{0}$, keeping in mind $(23)-(24)$

$$
\begin{aligned}
& \int_{\mathbb{D}}(\rho(\lambda, E))^{q-\alpha} d \sigma(\lambda)=(q-\alpha) \int_{0}^{2} y^{q-\alpha-1} \sigma(\{\lambda: \rho>y\}) d y \\
\geq & (q-\alpha) \int_{0}^{1} y^{q-\alpha-1} d y \int_{\Omega_{y}} \log \frac{1}{|\lambda|} d \mu_{0}(\lambda) \\
\geq & (q-\alpha) \int_{0}^{1} y^{q-\alpha-1}\left\{2^{-q}-1+q \int_{y}^{1} \frac{\nu_{E}(s)}{s^{q+1}} d s\right\} d y \\
= & q \int_{0}^{1} \frac{\nu_{E}(s)}{s^{\alpha+1}} d s+2^{-q}-1,
\end{aligned}
$$

and hence

$$
\int_{\mathbb{D}} \log \frac{1}{|\lambda|}(\rho(\lambda, E))^{q-\alpha} d \mu_{0}(\lambda)=+\infty
$$

To go over to (9) we note that in view of (12) and (13) the function $\rho^{-q}$ in the disk $\{z:|z|<1 / 2\}$ differs from $\int_{|\lambda|<1 / 2} \log (1 /|\lambda-z|) d \mu_{0}(\lambda)$ by a harmonic function. Hence, the latter integral is finite at $z=0$, and (9) follows. The proof is complete.

The following example shows that the result in Theorem 3 is optimal as well.

Example. Let $z_{n}=1-1 /(n+1), n=1,2, \ldots$ Consider an infinite canonical product (see, e.g., [12], p. 132)

$$
f(z):=\prod_{n=1}^{\infty} P\left(\frac{1-z_{n}^{2}}{1-z_{n} z}\right), \quad P(z)=(1-z) e^{z} .
$$

It follows from the Taylor expansion of $P$ that $|1-P(z)|<4|z|^{2}$ for $|z| \leq 2$. Since $\left|1-z_{n}^{2}\right|<2\left|1-z_{n} z\right|$ for $z \in \mathbb{D}$, we get

$$
\log |f(z)| \leq \sum_{n=1}^{\infty}\left|1-P\left(\frac{1-z_{n}^{2}}{1-z_{n} z}\right)\right|<4 \sum_{n=1}^{\infty}\left|\frac{1-z_{n}^{2}}{1-z_{n} z}\right|^{2} .
$$

Furthermore, for $z \in \mathbb{D}$

$$
\left|\frac{1-z_{n}^{2}}{1-z_{n} z}\right| \leq \frac{2}{|n(1-z)+1|} \leq \frac{2 \sqrt{2}}{n|1-z|+1}
$$

Hence for $n(z)=\left[|1-z|^{-1}\right]+1 \in \mathbb{N}$ the right hand side of (25) does not exceed

$$
32 n(z)+\frac{32}{|1-z|^{2}} \sum_{n=n(z)+1}^{\infty} \frac{1}{n^{2}} \leq \frac{64}{|1-z|}+\frac{32}{n(z)|1-z|^{2}} \leq \frac{96}{|1-z|} .
$$

So $f$ satisfies (10) with $E=\{1\}, q=1$, and the choice $\alpha=1-\varepsilon$ is optimal. 
The growth of certain canonical products in the unit disk was studied in $[8,9]$.

\section{Discrete SPECTRUM OF CONTRACTION OPERATORS}

The relation between the spectral theory of non-selfadjoint operators and the theory of analytic functions via perturbation determinants is well established due to the works of I. Gohberg, M. Krein, V. Matsaev and others. The variants of this approach were used recently in $[2,3]$.

The results from Section 2, in particular Theorem 3, can be applied to the operator theory in the same fashion as it is done in $[1,2,3]$.

Let $U$ be a unitary operator in the Hilbert space $\mathcal{H}, \sigma(U)=E$ its spectrum. The resolvent $R_{U}(z)=(U-z)^{-1}$ is an analytic in $\mathbb{D}$ operator-function, and (see, e.g., [14, Section V.3.8])

$$
\left\|R_{U}(z)\right\|=\rho^{-1}(z, \sigma(U)) \quad z \in \mathbb{D} .
$$

A bounded, linear operator $T$ in $\mathcal{H}$ is called a contraction if $\|T\| \leq 1$, $\|\cdot\|$ is the standard operator norm. Assume that $T-U$ is a compact operator. If $E \neq \mathbb{T}$, then by a general result from the perturbation theory $\sigma(T)=E \cup \sigma_{d}(T)$, where the discrete component of the spectrum $\sigma_{d}(T) \subset \overline{\mathbb{D}} \backslash E$ is at most countable set with all possible accumulation points in $E$, and each $z_{k} \in \sigma_{d}(T)$ is an eigenvalue of finite algebraic multiplicity.

Our goal here is to obtain some quantitative bound for the rate of convergence of $z_{k}$ to $E$ in the case, when $T-U \in \mathcal{S}_{q}, q>0$, the Schatten-von Neumann classes of compact operators, i.e., the sequence of eigenvalues of the operator $|T-U|$ belongs to $\ell^{q}$. The norm in $\mathcal{S}_{q}$ is denoted by $\|\cdot\|_{q}$, and the standard operator norm $\|\cdot\|=\|\cdot\|_{\infty}$. The family $\left\{\mathcal{S}_{q}\right\}$ is known to be nested: $\mathcal{S}_{q_{1}} \subset \mathcal{S}_{q_{2}}$ for $0<q_{1}<q_{2} \leq \infty$, and $\|A\|_{q_{2}} \leq\|A\|_{q_{1}}$. If $A \in \mathcal{S}_{q}, q \geq 1$, and $S$ is a bounded linear operator then $\overline{A S} \in \mathcal{S}_{q}$ and $\|A S\|_{q} \leq\|A\|_{q}\|S\|$. An extensive information on the subject is Gohberg-Krein [10], Simon [19], and Dunford-Schwartz [5, Chapter XI.9].

The key analytic tool is the so called renormalized determinant $\operatorname{det}_{n}(I+A)$, defined for $A \in \mathcal{S}_{n}$ and $n=1,2, \ldots$ In particular, we need the following continuity property (cf. [19, Theorem 9.2, (c)])

$\left|\operatorname{det}_{n}(I+A)-\operatorname{det}_{n}(I+B)\right| \leq\|A-B\|_{n} \exp \left(C_{n}\left(1+\|A\|_{n}^{n}+\|B\|_{n}^{n}\right)\right)$,

a constant $C_{n}$ depends only on $n$. If $T-U \in \mathcal{S}_{n}$, then the perturbation determinant

$$
u_{n}(z):=\operatorname{det}_{n}(T-z)(U-z)^{-1}=\operatorname{det}_{n}\left(I+(T-U)(U-z)^{-1}\right)
$$

is a well defined and analytic function in $\mathbb{D}$, and its zero set in $\mathbb{D}$ is $Z_{u_{n}}=\sigma_{d}(T) \cap \mathbb{D}$, with the order of each zero equal the algebraic multiplicity of the eigenvalue. 
Theorem 4. Let $U$ be a unitary operator in $\mathcal{H}, \sigma(U)=E \neq \mathbb{T}$, and $I(\alpha, E)<\infty$ in (4) for some $\alpha \in \mathbb{R}$. Let a contraction $T$ be a $\mathcal{S}_{q^{-}}$ perturbation of $U: T-U \in \mathcal{S}_{q}, q>0$. Then

$$
\sum_{z \in \sigma_{d}(T)}(1-|z|) \rho(z, E)^{(q-\alpha)_{+}}<\infty
$$

Moreover, there is a constant $\delta=\delta_{q}, 0<\delta_{q}<1$, which depends only on $q$, such that for $\|T-U\|_{q}<\delta_{q}$ the inequality holds

$$
\sum_{z \in \sigma_{d}(T)}(1-|z|) \rho(z, E)^{(q-\alpha)_{+}} \leq C(q, E) \max \left(\|T-U\|_{q},\|T-U\|_{q}^{q}\right)
$$

Remark. Obviously, the eigenvalues of $T$ with $|z|=1$ do not enter the left hand side, so we claim nothing about this part of the discrete spectrum. However, this part is missing as long as $T$ is a completely non-unitary contraction.

Proof. Take a positive integer $n$ from $q \leq n<q+1$, so $T-U \in \mathcal{S}_{n}$. By [5, Lemma XI.9.22,(d)]

$$
\begin{aligned}
\left|u_{n}(z)\right| & \leq \exp \left(c_{q}\left\|(T-U)(U-z)^{-1}\right\|_{q}^{q}\right) \\
& \leq \exp \left(c_{q}\|T-U\|_{q}^{q}\left\|(U-z)^{-1}\right\|^{q}\right) \\
& \leq \exp \left(c_{q}\|T-U\|_{q}^{q} \rho^{-q}(z, E)\right) .
\end{aligned}
$$

An application of the first statement of Theorem 3 proves (27).

Throughout the rest of the proof $c_{q}$ stands for different positive constants which depend only on $q$. Since $\|T-U\|<1$, then $T$ is invertible, and

$$
u_{n}(0)=\operatorname{det}_{n}\left(I+(T-U) U^{-1}\right) \neq 0 .
$$

Put $f(z)=u_{n}(z) / u_{n}(0),|f(0)|=1$, so that

$$
\log |f(z)| \leq \frac{c_{q}\|T-U\|_{q}^{q}}{\rho^{q}(z, E)}+\log \frac{1}{\left|u_{n}(0)\right|} .
$$

Bound (26) with $A=(T-U) U^{-1}, B=0$ gives in view of the monotonicity of norms

$$
\left|u_{n}(0)-1\right| \leq\|T-U\|_{q} \exp \left(C_{q}^{\prime}\left(1+\|T-U\|_{q}^{n}\right)\right)<\frac{1}{2}
$$

as soon as $\delta_{q}$ is small enough. Hence

$$
\log \frac{1}{\left|u_{n}(0)\right|} \leq \log 4\left|u_{n}(0)-1\right| \leq c_{q}\|T-U\|_{q} .
$$

An application of the second statement of Theorem 3 gives (28). 


\section{REFERENCES}

[1] A. Borichev, L. Golinskii, and S. Kupin, A Blaschke-type condition and its application to complex Jacobi matrices, preprint arXive:0712.0407v2.

[2] M. Demuth, G. Katriel, Eigenvalue inequalities in terms of Schatten norm bounds on differences of semigroups, and application to Schrödinger operators, Annales Henri Poincaré, to appear.

[3] M. Demuth, M. Hansmann, and G. Katriel, On the distribution of eigenvalues of non-selfadjoint operators, preprint arXive:0802.2468v1.

[4] M. M. Djrbashian, Theory of Factorization of Functions Meromorphic in the Disk. In: Proceedings of the ICM, Vancouver, B.C., 1974, vol. 2, 197-202, USA 1975.

[5] N. Dunford, J.T. Schwartz, Linear Operators. Part II: Spectral Theory. Selfadjoint Operators in Hilbert Space, John Wiley and Sons (New York-London), 1963.

[6] K. Falconer, Fractal Geometry. Mathematical Foundations and Applications. John Wiley and Sons, Chichester, New York, 1990.

[7] J. Garnett, Bounded analytic functions. Graduate Texts in Mathematics, vol. 236. Springer, New York, 2007.

[8] M.Girnyk, On asymptotic properties of certain canonical products. Sib.Math.J., v.15, No 5, (1974), 1036-1048.

[9] M.Girnyk, On asymptotic properties of certain canonical products, II. Sib.Math.J., v.17, No 5, (1976), 967-985.

[10] I. Gohberg, M. Krein, Introduction to the Theory of Linear Nonselfadjoint Operators. Translations of AMS, vol. 18, AMS, Providence, 1969.

[11] W.K. Hayman, B. Korenblum, A critical growth rate for functions regular in a disk. Michigan Math. J. 27 (1980), 21-30.

[12] H. Hedenmalm, B. Korenblum, K. Zhu, Theory of Bergman spaces. Graduate Texts in Mathematics, vol. 199. Springer-Verlag, New York, 2000.

[13] A. M. Jerbashian, On the theory of weighted classes of area integrable regular functions. Complex Variables, 50 (2005), 155-183.

[14] T. Kato, Perturbation Theory for Linear Operators. Springer-Verlag, Berlin, 1966.

[15] E. Lieb, M. Loss, Analysis. Graduate Studies in Mathematics, vol. 14, AMS, Providence, RI, 1997.

[16] V.I Matsaev, E.Z.Mogulskii, Theorem on division for analytic functions with a given majorant and some applications, Zap. Nauchn. Sem. LOMI (1976), V.56, 72-89 (Russian).

[17] T. Ransford, Potential Theory in the Complex Plane. London Math. Soc. Student Texts, vol. 28, Cambridge University Press, 1995.

[18] F. A. Shamoyan, On zeros of analytic in the disc functions growing near its boundary. Journal of Contemporary Mathematical Analysis (Armenian Academy of Sciences) vol. 18, no. 1, 1983.

[19] B. Simon, Trace ideals and their applications. Mathematical Surveys and Monographs, vol. 120. AMS, Providence, RI, 2005.

Mathematical School, Kharkov National University, Swobody sq.4, KHARKOV, 61077 UKRAINE

E-mail address: Sergey.Ju.Favorov@univer.kharkov.ua

Mathematics Division, Institute for Low Temperature Physics and Engineering, 47 Lenin aVe., Kharkov 61103, Ukraine

E-mail address: leonid.golinskii@gmail.com 